\title{
Electron scattering times in ZnO based polar heterostructures
}

Cite as: Appl. Phys. Lett. 107, 082102 (2015); https://doi.org/10.1063/1.4929381

Submitted: 17 June 2015 . Accepted: 06 August 2015 . Published Online: 24 August 2015

J. Falson, Y. Kozuka, J. H. Smet, T. Arima, A. Tsukazaki, and M. Kawasaki
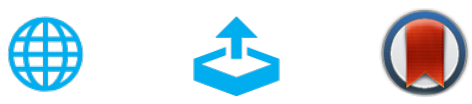

View Online

Export Citation

\section{ARTICLES YOU MAY BE INTERESTED IN}

Challenges and opportunities of ZnO-related single crystalline heterostructures

Applied Physics Reviews 1, 011303 (2014); https://doi.org/10.1063/1.4853535

Optical probing of $\mathrm{MgZnO} / \mathrm{ZnO}$ heterointerface confinement potential energy levels Applied Physics Letters 106, 082102 (2015); https://doi.org/10.1063/1.4913313

Alloy disorder modulated electron transport at $\mathrm{Mg}_{\mathrm{x}} \mathrm{Zn}_{1-\mathrm{x}} \mathrm{O} / \mathrm{ZnO}$ heterointerface AIP Advances 7, 015029 (2017); https://doi.org/10.1063/1.4974462

Lock-in Amplifiers Find out more today

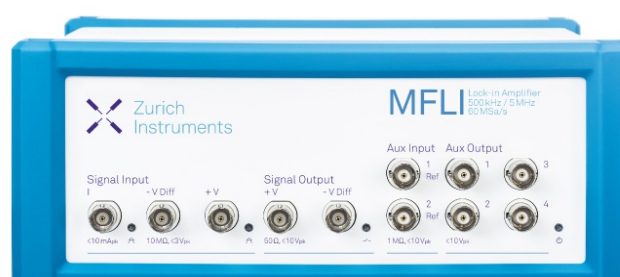

- Zurich - Instruments 


\title{
Electron scattering times in $\mathrm{ZnO}$ based polar heterostructures
}

\author{
J. Falson, ${ }^{1,2,3, a)}$ Y. Kozuka, ${ }^{1}$ J. H. Smet, ${ }^{3}$ T. Arima, ${ }^{2,4}$ A. Tsukazaki, ${ }^{5,6}$ and M. Kawasaki ${ }^{1,4}$ \\ ${ }^{1}$ Department of Applied Physics and Quantum-Phase Electronics Center (QPEC), The University of Tokyo, \\ Tokyo 113-8656, Japan \\ ${ }^{2}$ Department of Advanced Materials Science, The University of Tokyo, Kashiwa 277-8561, Japan \\ ${ }^{3}$ Max Planck Institute for Solid State Research, D-70569 Stuttgart, Germany \\ ${ }^{4}$ RIKEN Center for Emergent Matter Science (CEMS), Wako 351-0198, Japan \\ ${ }^{5}$ Institute for Materials Research, Tohoku University, Sendai 980-8577, Japan \\ ${ }^{6}$ PRESTO, Japan Science and Technology Agency (JST), Tokyo 102-0075, Japan
}

(Received 17 June 2015; accepted 6 August 2015; published online 24 August 2015)

\begin{abstract}
The remarkable historic advances experienced in condensed matter physics have been enabled through the continued exploration and proliferation of increasingly richer and cleaner material systems. In this work, we report on the scattering times of charge carriers confined in state-of-theart $\mathrm{MgZnO} / \mathrm{ZnO}$ heterostructures displaying electron mobilities in excess of $10^{6} \mathrm{~cm}^{2} / \mathrm{V} \mathrm{s}$. Through an examination of low field quantum oscillations, we obtain the effective mass of charge carriers, along with the transport and quantum scattering times. These times compare favorably with high mobility $\mathrm{AlGaAs} / \mathrm{GaAs}$ heterostructures, suggesting the quality of $\mathrm{MgZnO} / \mathrm{ZnO}$ heterostructures now rivals that of traditional semiconductors. (C) 2015 AIP Publishing LLC.
\end{abstract}

[http://dx.doi.org/10.1063/1.4929381]

Two-dimensional electron systems (2DES) are known to display exceptionally high conductivity at low temperatures where the effect of phonon scattering is negligible. The low temperature Drude conductivity, $\sigma=e n \mu$, where $e$ is the elementary charge and $n$ is the 2DES charge density, includes the electron mobility $\mu$ that is determined by elastic scattering events. In the case of the AlGaAs/GaAs 2DES, ultrahigh mobilities in excess of $3 \times 10^{7} \mathrm{~cm}^{2} / \mathrm{V}$ s have been achieved through the mastering of modulation doping techniques, which place charged dopants away from the 2DEShosting quantum well. ${ }^{1,2}$ What ultimately limits the mobility is considered to be the presence of background impurities unintentionally incorporated during the crystal growth process. $^{3}$ With this knowledge, it may seem a foregone conclusion that these samples represent the highest "quality" platforms for investigating the rich quantum transport phenomenon observed in the 2DES at low temperatures. Such conclusions are however premature, as the raw mobility value takes into account the effective mass $m^{*}$ of charge carriers. Thus, it is less relevant to judge sample quality and in many cases a fairer comparison of the "cleanness" of different materials can be achieved through comparing the transport scattering time of electrons, $\tau_{\mathrm{tr}}=\mu m^{*} / e$. In terms of the in-plane momentum, $q=2 k_{\mathrm{F}} \sin (\phi / 2)$ transferred during an individual scattering event, the momentum relaxation rate is given by ${ }^{4,5}$

$$
\frac{1}{\tau_{\mathrm{tr}}}=\frac{m^{*}}{\hbar^{3}} \int_{0}^{2 \pi} \frac{d \phi}{2 \pi} W(q)(1-\cos \phi) .
$$

Here, $\hbar$ is the reduced Planck constant, $k_{\mathrm{F}}=(2 \pi n)^{1 / 2}$ is the Fermi wave vector, $W(q)$ is the Fourier component of the correlation function of screened disorder potential in the plane of 2DES, and $\phi$ is the scattering angle.

\footnotetext{
${ }^{a)}$ Electronic mail: j.falson@fkf.mpg.de
}

The factor $(1-\cos \phi)$ in Eq. (1) reflects the fact that small-angle scattering with $\cos \phi \ll 1$ is inefficient for backscattering and hence $\tau_{\text {tr }}$ is weighted towards large-angle scattering events. It however remains a poignant fact that even $\tau_{\text {tr }}$ on its own cannot predict the "quality" of a 2DES. Indeed, the quality of transport, for example, given by the activation energy of fragile fractional quantum Hall states, often shows a vague correlation with the $\tau_{\text {tr }}$ of the sample. ${ }^{2,6-8}$ This apparent contradiction has spurred theoretical efforts to pin-point a parameter beyond $\tau_{\text {tr }}$ which can account for the discrepancy. ${ }^{9,10}$ Attention has turned to the quantum scattering time, $\tau_{\mathrm{q}}$, which is expressed in the form (1) with the factor $(1-\cos \phi)$ replaced by unity. It implies that large and small-angle scattering events in the quantum rate $\tau_{\mathrm{q}}^{-1}$ are equally weighed (1). Experimentally, $\tau_{\mathrm{q}}$ may be quantified through an examination of the broadening of Landau levels, for example, through the analysis of low field Shubnikov-de Haas $(\mathrm{SdH})$ oscillations. That said, the correlation between this parameter and quality, at least in the AlGaAs/GaAs 2DES, remains clouded. ${ }^{2,6}$

In this work, we present data for state-of-the-art polar $\mathrm{ZnO}$ heterostructures. ${ }^{11} \mathrm{We}$ explore quantitatively the transport and quantum scattering times of $2 \mathrm{D}$ electrons confined at the $\mathrm{MgZnO} / \mathrm{ZnO}$ heterointerface with the motivation of gauging the current state of growth technology and facilitating a more complete intermaterial comparison. The $\mathrm{MgZnO} /$ $\mathrm{ZnO}$ system has a number of distinct features. ${ }^{11}$ Most notably, there are no intentional remote doping structures within the heterostructure which donate electrons, in contrast to the modulation doped AlGaAs/GaAs heterostructures used to achieve record mobilities. ${ }^{1,2}$ Rather the 2DES forms as a result of the discontinuity in the electric polarization at the interface of the capping $\left(\mathrm{Mg}_{x} \mathrm{Zn}_{1-x} \mathrm{O}\right)$ and buffer $(\mathrm{ZnO})$ layers, where $x$ is a growth controlled parameter. The doped $\mathrm{Mg}$ atoms remain isovalent with the bulk and rather induce the 2DES by producing an elastic strain that modifies the 
internal polarization field. The $x$ content is known ${ }^{12}$ to have a strong impact on the mobility (i.e., $\tau_{\mathrm{tr}}$ ) of the 2DES. The highest mobility reported thus far occurs at $x \approx 0.01$ $\left(n \approx 1.5 \times 10^{11} \mathrm{~cm}^{-2}\right) .{ }^{12}$ At either lower or higher charge densities, the mobility limiting mechanism is considered to be background impurity and interface roughness scattering, respectively. Finally, despite the modest mobility of electrons of about $5 \times 10^{5} \mathrm{~cm}^{2} / \mathrm{V} \mathrm{s}$, the ability to observe exotic ground states such as even-denominator fractional quantum Hall states has recently been demonstrated. ${ }^{13}$ This observation alone necessitates the quantitative exploration we present.

Low temperature magnetotransport measurements were performed on a series of $\mathrm{Mg}_{x} \mathrm{Zn}_{1-x} \mathrm{O} / \mathrm{ZnO}$ heterostructures grown by ozone assisted molecular beam epitaxy with varying $x$. The record mobility $\left(\mu>10^{6} \mathrm{~cm}^{2} / \mathrm{V}\right.$ s) has been enabled through a study of the stoichiometry of samples grown at various growth conditions as well as of impurity sources, the details of which will be published elsewhere. As for substrate treatment, polished $\mathrm{Zn}$-polar single crystal $\mathrm{ZnO}$ substrates (Tokyo Denpa Co.) were prepared through $30 \mathrm{~s}$ $\mathrm{HCl}$ etching, as described in Ref. 14. All samples have nominally the same growth conditions; moderately $\mathrm{Zn}$ rich flux ratios with a growth rate of $600-800 \mathrm{~nm} / \mathrm{h}$. Surface roughness of the films is less than $0.1 \mathrm{~nm}$. Physical characteristics of the films resemble those reported in Ref. 15. The mobility is calculated through low temperature Hall measurements and the sheet resistivity. For the examination of SdH oscillations, standard low-frequency lock-in measurements in a dilution refrigerator (base $T \approx 40 \mathrm{mK}$ ) were performed. All samples were measured in van der Pauw geometry with a device size of roughly $3 \times 3 \mathrm{~mm}$ cut from raw wafers and contacted by soldered indium.

Figure 1 displays the temperature dependence ( $T=40-100 \mathrm{mK}$ ) of low field magnetotransport for a sample of $n=1.6 \times 10^{11} \mathrm{~cm}^{-2}$ and $\mu=1200000 \mathrm{~cm}^{2} / \mathrm{V} \mathrm{s}$ as a function of perpendicular magnetic field $(B)$. This low temperature is required for two reasons. First, the mobility of dilute heterostructures saturates well below $500 \mathrm{mK}$ (Refs. 12 and 16) and second, the oscillations are quickly dampened when moving to higher $T$, as can be seen in the data. The $\mathrm{SdH}$ oscillations are resolved for a magnetic field as low as $B \approx 0.085 \mathrm{~T}$ with the spin splitting of oscillations occurring at around $B \approx 0.3 \mathrm{~T}$. For the analysis, we rotate the sample in-situ until the cyclotron and Zeeman energies coincide. ${ }^{17}$ Gaps, therefore, represent the cyclotron energy and we analyze the data according to the Dingle expression, ${ }^{18}$

$$
\frac{\Delta R_{x x}}{R_{0}}=\frac{4 \zeta}{\sinh \zeta} e^{-\pi / \omega_{\mathrm{c}} \tau_{\mathrm{q}}}, \quad \zeta=\frac{2 \pi^{2} k_{\mathrm{B}} T}{\hbar \omega_{\mathrm{c}}} .
$$

Here, $\Delta R_{x x} / R_{0}$ is the amplitude of oscillations normalized by the resistance at zero field and $\omega_{\mathrm{c}}=e B / \mathrm{m}^{*}$ is the cyclotron frequency, with $m^{*}$ the effective mass of charge carriers. Figs. 1(b) and 1(c) show the representative analyses for the heterostructure presented. We note in panel $\mathrm{c}$ the plot of $\frac{\Delta R_{x x}}{R_{0}} \frac{\sinh \zeta}{\zeta}$ vs. $1 / B$ through the experimental points produces the correct intercept of 4, as expected from Eq. (1). We represent the data obtained through this method as solid points in Figs. 2 and 3 and term them $\tau_{\mathrm{q}, \text { Dingle. For the }}$
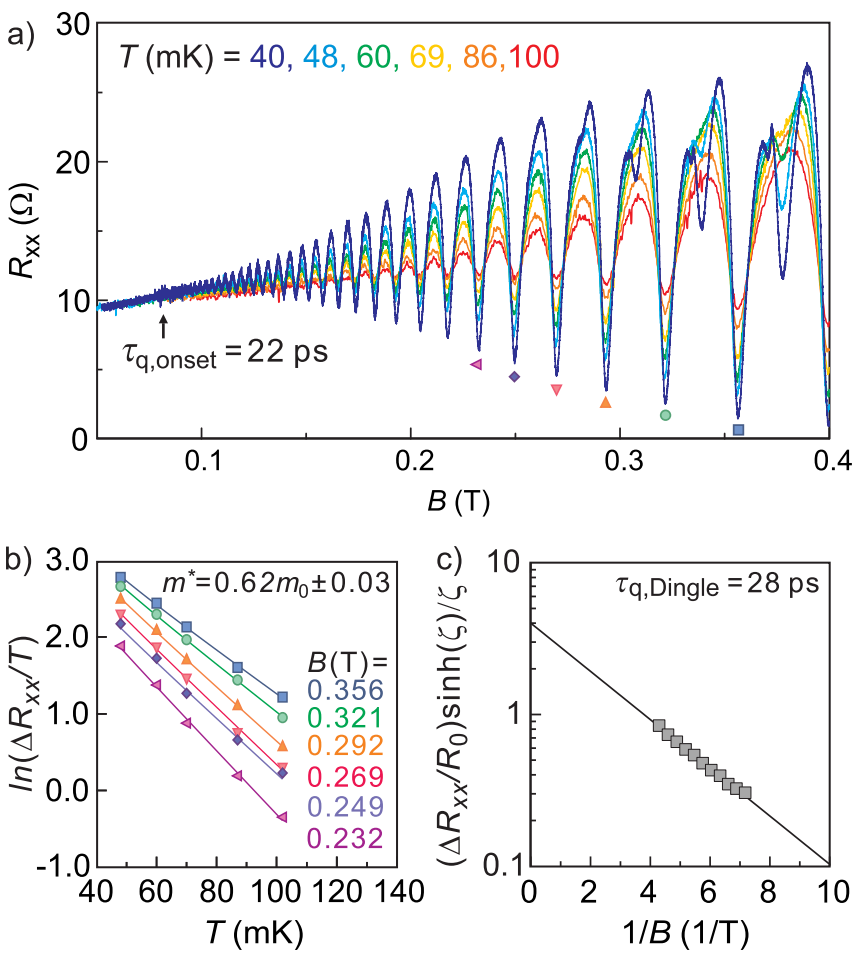

FIG. 1. Analysis of Shubnikov-de Haas oscillations of a sample $n=1.6$ $\times 10^{11} \mathrm{~cm}^{-2}$ and $\mu=1200000 \mathrm{~cm}^{2} / \mathrm{V} \mathrm{s}$ as a function of $B$ where the sample is titled $\theta=54^{\circ}$ to the coincidence position. (a) Temperature dependent low field magnetotransport which permits the deduction of (b) the effective mass $\left(m^{*}=0.62 m_{0} \pm 0.03\right)$, and (c) quantum scattering time $\left(\tau_{\mathrm{q}}=28 \mathrm{ps}\right)$.

heterostructure shown, the analysis reveals that $m^{*}$ is significantly enhanced $\left(m^{*}=0.62 m_{0} \pm 0.03, m_{0}\right.$ is the bare electron mass) over the bulk band mass, $m_{\mathrm{b}}^{*}=0.3 m_{0}$. This mass enhancement is attributed to electron-electron interactioninduced parameter renormalization within the Fermi-liquid theory, and is similarly seen in other strongly interacting 2DES. ${ }^{19}$ The Dingle analysis could not be applied for charge
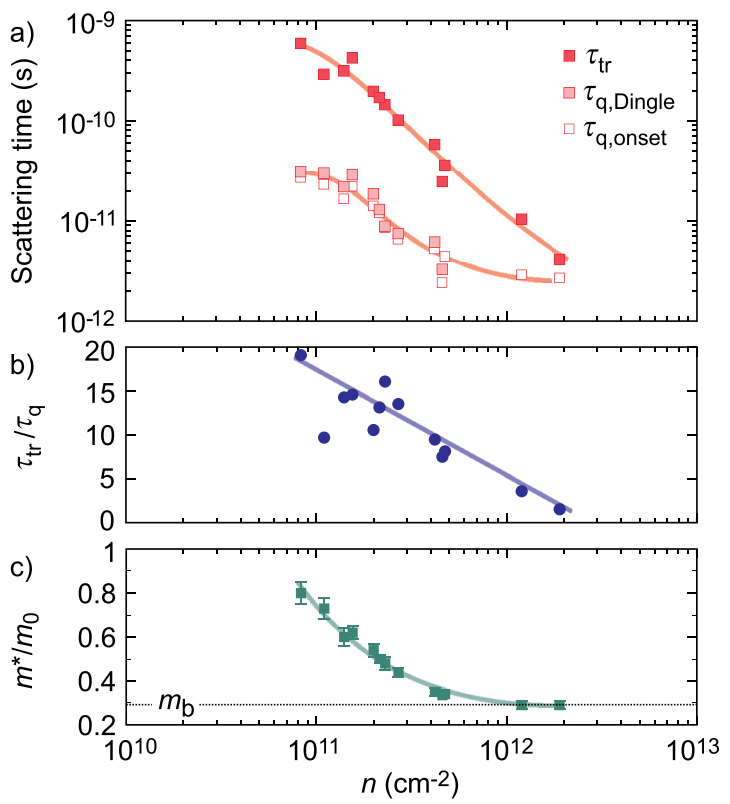

FIG. 2. Experimentally determined (a) $\tau_{\mathrm{tr}}, \tau_{\mathrm{q}}$ and (b) $\tau_{\mathrm{tr}} / \tau_{\mathrm{q}}$, calculated from $\tau_{\mathrm{q}, \text { Dingle }}$ where available, and (c) $\mathrm{m}^{*} / \mathrm{m}_{0}$ as a function of $n$. Coloured lines are visual guides. 

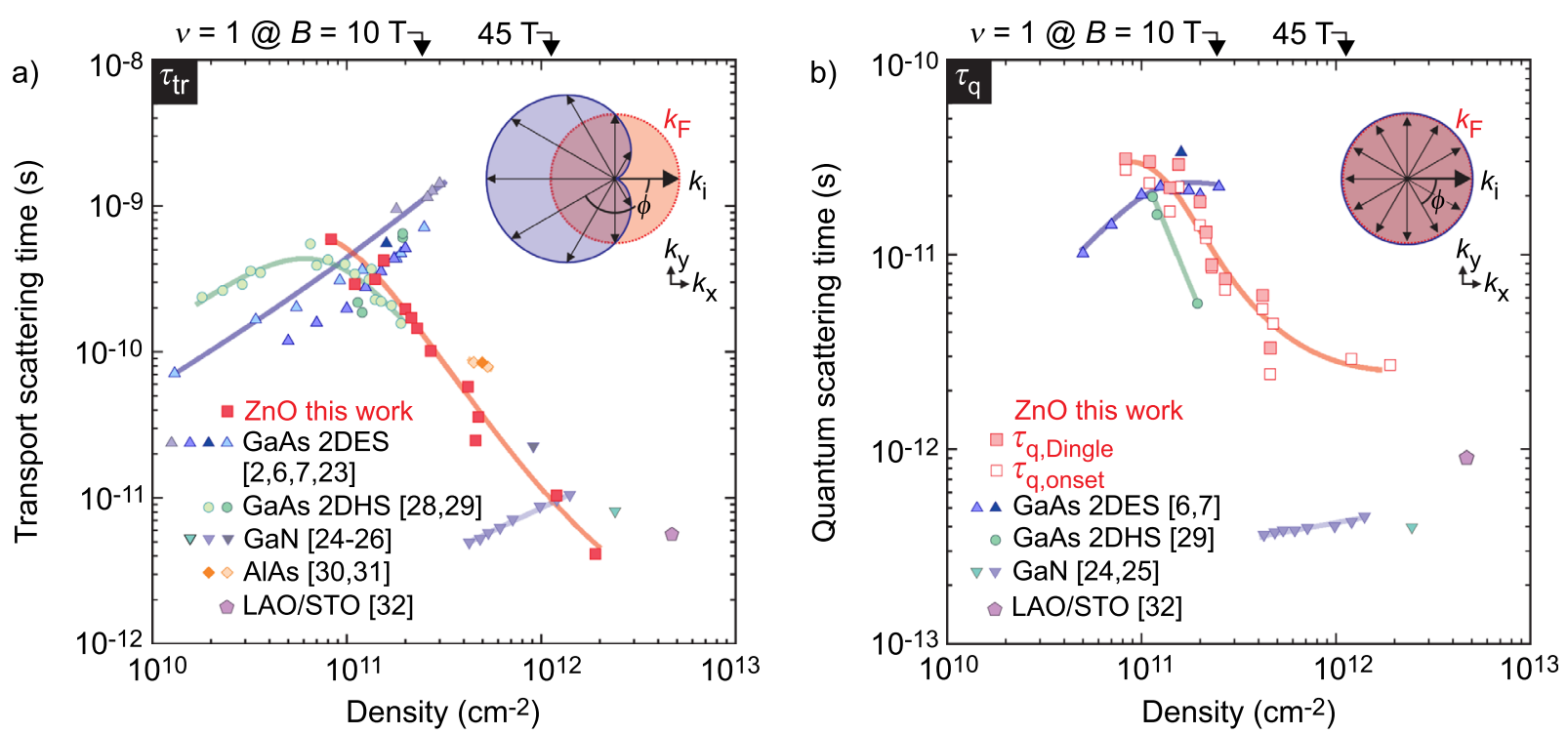

FIG. 3. An intermaterial comparison of (a) $\tau_{\text {tr }}$ and (b) $\tau_{\mathrm{q}}$. Data are taken from AlGaAs/GaAs $2 \mathrm{DES},{ }^{2,67,23} \mathrm{AlGaAs} / \mathrm{GaAs} 2 \mathrm{DHS},{ }^{28,29} \mathrm{AlGaN} / \mathrm{GaN},{ }^{24-26} \mathrm{AlGaAs} /$ $\mathrm{AlAs},{ }^{30,31}$ and $\mathrm{LaAlO}_{3} / \mathrm{SrTiO}_{3}{ }^{32}$ The insets schematically represent the Fermi circle (pink shading) with the weighting factors (blue shading) which distinguish transport and quantum scattering rates. The upper arrows indicate the charge density where the $\nu=1$ quantum Hall state $\left(\nu=\frac{h n}{e B}\right)$ occurs for $B=10$ and $45 \mathrm{~T}$.

density $n<8 \times 10^{10} \mathrm{~cm}^{-2}$. This is due to increasingly acute dampening of the oscillations even at $T<100 \mathrm{mK}$, despite the high mobility. This is presumably a result of the enhanced effective mass suppressing the cyclotron gap. We note that the low temperature also reduces the relevance of inelastic collision mediated effects on $\tau_{\mathrm{q}}{ }^{20}$

A second approach to estimating $\tau_{\mathrm{q}}$ is via the onset magnetic field of $\mathrm{SdH}$ oscillations through the relationship, $\tau_{\mathrm{q}, \text { onset }}^{-1}=2 \omega_{\mathrm{c}, \text { onset }}$ where $\omega_{\mathrm{c}, \text { onset }}$ is the cyclotron frequency at the start of oscillations. This analysis may be performed as close as possible to zero field and therefore circumvents complications associated with spin splitting and the onset of the quantum Hall effect. Overall, a good agreement is achieved between these two methods for obtaining $\tau_{\mathrm{q}}$ as can be seen when comparing Figs. 1(a) and 1(c), as well as the data in Fig. 2(a) where we plot the values extracted by this technique as open symbols. Generally, a slightly reduced number is obtained due to some ambiguity associated with determining the true onset amongst finite signal noise.

Figure 2 plots in panels (a): $\tau_{\text {tr }}$ and $\tau_{\mathrm{q}}$, (b): the ratio $\tau_{\mathrm{tr}} /$ $\tau_{\mathrm{q}}$, and (c): $m^{*}$ as a function of $n$. The experimentally determined effective mass is used for calculations of both $\tau_{\text {tr }}$ and $\tau_{\mathrm{q}}$. The values of $m^{*}$ for the high density regime agree well with Ref. 17 where interaction mediated renormalization effects are weak. Beginning in this high density regime, both $\tau_{\mathrm{tr}}$ and $\tau_{\mathrm{q}}$ are suppressed and take on a ratio $\tau_{\mathrm{tr}} / \tau_{\mathrm{q}} \approx 1$. This implies scattering which is not predominantly small angle as expected when short range alloy of interface roughness mechanisms is prevalent when the wavefunction narrows and the quantum well deepens. ${ }^{16}$ When reducing the density down to $n \approx 1 \times 10^{11} \mathrm{~cm}^{-2}$, both $\tau_{\mathrm{tr}}$ and $\tau_{\mathrm{q}}$ are enhanced. In this regime, $\tau_{\text {tr }} \approx 600$ ps and $\tau_{\mathrm{q}} \approx 30 \mathrm{ps}$. We note that the enhanced $m^{*}$ obtained through the analysis of SdH increases both of these values. However, even with the use of the band mass $m^{*}=0.3 m_{0}$ the deduced $\tau_{\mathrm{q}}$ exceeds $10 \mathrm{ps}$ while producing a worse Dingle plot. Notably, a ratio of $\tau_{\mathrm{tr}} / \tau_{\mathrm{q}} \approx 100$ is not encountered. Such a ratio is a feature of modulation-doped $\mathrm{AlGaAs} / \mathrm{GaAs}$ heterostructures, where $\tau_{\text {tr }}$ is limited by background impurities while $\tau_{\mathrm{q}}$ is determined by the smoothly varying potential background imposed by the remote dopants. ${ }^{10}$ Rather, $\tau_{\mathrm{tr}} / \tau_{\mathrm{q}}$ approaches $\approx 20$ when delving into more dilute samples and effects of interface roughness are mitigated. ${ }^{16}$ This final value may be within the realms of homogeneous background impurity limited scattering times, ${ }^{21,22}$ but we cannot rule out other potential origins such as the sample surface which is on the order of $500 \mathrm{~nm}$ away from the 2DES.

In Fig. 3, we present an intermaterial comparison of high quality samples reported within the literature. We limit the range of materials to those with a parabolic electron dispersion and thus exclude graphene. The insets illustrate the scattering angle dependence of the weighting factors [panel (a) $(1-\cos \phi)$ for $\tau_{\mathrm{tr}}^{-1}$, see Eq. (1), and panel (b) unity for $\left.\tau_{\mathrm{q}}^{-1}\right]$, which distinguishes the rates $\tau_{\mathrm{tr}}^{-1}$ and $\tau_{\mathrm{q}}^{-1}$ within the simplest semiclassical picture. While the $\mathrm{AlGaAs} / \mathrm{GaAs}^{2,6,7,23}$ and $\mathrm{AlGaN} / \mathrm{GaN}^{24-26}$ 2DES show enhanced scattering times with increasing density due to more effective screening of background impurities ${ }^{3}$ and charged dislocations, ${ }^{27}$ respectively, the scattering times in the $\mathrm{Mg}_{x} \mathrm{Zn}_{1-x} \mathrm{O} / \mathrm{ZnO}$ 2DES are suppressed due to enhanced alloy scattering for the same range of densities. ${ }^{16}$ In the dilute regime $\left(n \approx 1 \times 10^{11} \mathrm{~cm}^{-2}\right)$, the data set conveys that in terms of both $\tau_{\mathrm{tr}}$ and $\tau_{\mathrm{q}}$, scattering times in $\mathrm{MgZnO} / \mathrm{ZnO}$ heterostructures are comparable with high quality $\mathrm{AlGaAs} / \mathrm{GaAs}$ heterostructures with mobility on the order of $10^{7} \mathrm{~cm}^{2} / \mathrm{Vs}$ which show fragile fractional quantum Hall features, such as the $\nu=5 / 2$ state. $^{2,6,7}$ Along with the AlGaAs/GaAs two-dimensional hole system (2DHS), ${ }^{28,29}$ these three platforms stand out as the highest quality available, ahead of the AlGaAs/AlAss, ${ }^{30,31} \mathrm{AlGaN} / \mathrm{GaN},{ }^{24-26}$ and $\mathrm{LaAlO}_{3} / \mathrm{SrTiO}_{3},{ }^{32}$ although recent progress is being made for this latter system. ${ }^{33}$

Finally, we indicate the charge density where the $\nu=1$ quantum Hall state $\left(\nu=\frac{h n}{e B}\right)$ occurs for $B=10$ and $45 \mathrm{~T}$. Importantly, the longest scattering time $\mathrm{MgZnO} / \mathrm{ZnO}$ samples reside below the $B=10 \mathrm{~T}$ mark which represents fields typically available in laboratory environments. The 
observation of fragile fractional quantum Hall physics until recently was the exclusive privilege of modulation doped semiconductor 2DES. With $\mathrm{ZnO}$, a system has become available in which scattering is governed by different mechanisms. It thereby discloses the possibility of exploring alternate facets of the correlation between scattering times and the rich set of ground states that may form in a $2 \mathrm{DES}$ as the magnetic field is tuned.

We acknowledge valuable discussions with D. Maryenko, I. Dmitriev, M. Manfra, and G. Csathy. This work was partly supported by JSPS Grant-in-Aid for Scientific Research(S) No. 24226002 and for Young Scientist (A) No. 23686008 and the "Funding Program for World-Leading Innovative R\&D on Science and Technology (FIRST Program)," initiated by the Council for Science and Technology Policy (CSTP). This work was carried out by joint research of the Cryogenic Research Center, the University of Tokyo.

${ }^{1}$ L. Pfeiffer and K. West, Physica E 20, 57 (2003).

${ }^{2}$ V. Umansky, M. Heiblum, Y. Levinson, J. Smet, J. Nubler, and M. Dolev, J. Cryst. Growth 311, 1658 (2009).

${ }^{3}$ E. H. Hwang and S. Das Sarma, Phys. Rev. B 77, 235437 (2008).

${ }^{4}$ T. Ando, A. B. Fowler, and F. Stern, Rev. Mod. Phys. 54, 437 (1982).

${ }^{5}$ I. A. Dmitriev, A. D. Mirlin, D. G. Polyakov, and M. A. Zudov, Rev. Mod. Phys. 84, 1709 (2012).

${ }^{6}$ J. Nuebler, V. Umansky, R. Morf, M. Heiblum, K. von Klitzing, and J. Smet, Phys. Rev. B 81, 035316 (2010).

${ }^{7}$ C. R. Dean, B. A. Piot, P. Hayden, S. Das Sarma, G. Gervais, L. N. Pfeiffer, and K. W. West, Phys. Rev. Lett. 100, 146803 (2008).

${ }^{8}$ T. Sajoto, Y. W. Suen, L. W. Engel, M. B. Santos, and M. Shayegan, Phys. Rev. B 41, 8449 (1990).

${ }^{9}$ S. Das Sarma and F. Stern, Phys. Rev. B 32, 8442 (1985).

${ }^{10}$ S. Das Sarma and E. H. Hwang, Phys. Rev. B 90, 035425 (2014).

${ }^{11}$ Y. Kozuka, A. Tsukazaki, and M. Kawasaki, Appl. Phys. Rev. 1, 011303 (2014).

${ }^{12}$ J. Falson, D. Maryenko, Y. Kozuka, A. Tsukazaki, and M. Kawasaki, Appl. Phys. Express 4, 091101 (2011).

${ }^{13}$ J. Falson, D. Maryenko, B. Friess, D. Zhang, Y. Kozuka, A. Tsukazaki, J. H. Smet, and M. Kawasaki, Nat. Phys. 11, 347 (2015).
${ }^{14}$ S. Akasaka, K. Nakahara, H. Yuji, A. Tsukazaki, A. Ohtomo, and M. Kawasaki, Appl. Phys. Express 4, 035701 (2011).

${ }^{15}$ Y. Kozuka, J. Falson, Y. Segawa, T. Makino, A. Tsukazaki, and M. Kawasaki, J. Appl. Phys. 112, 043515 (2012).

${ }^{16}$ Q. Li, J. Zhang, J. Chong, and X. Hou, Appl. Phys. Express 6, 121102 (2013).

${ }^{17}$ A. Tsukazaki, A. Ohtomo, M. Kawasaki, S. Akasaka, H. Yuji, K. Tamura, K. Nakahara, T. Tanabe, A. Kamisawa, T. Gokmen, J. Shabani, and M. Shayegan, Phys. Rev. B 78, 233308 (2008).

${ }^{18}$ P. Coleridge, Phys. Rev. B 44, 3793 (1991).

${ }^{19}$ S. V. Kravchenko and M. P. Sarachik, Rep. Prog. Phys. 67, 1 (2004).

${ }^{20}$ Y. Adamov, I. V. Gornyi, and A. D. Mirlin, Phys. Rev. B 73, 045426 (2006).

${ }^{21}$ S. MacLeod, K. Chan, T. Martin, A. Hamilton, A. See, A. Micolich, M. Aagesen, and P. Lindelof, Phys. Rev. B 80, 035310 (2009).

${ }^{22}$ A. Gold, J. Appl. Phys. 110, 043702 (2011).

${ }^{23}$ V. Umansky, R. de Picciotto, and M. Heiblum, Appl. Phys. Lett. 71, 683 (1997).

${ }^{24}$ E. Frayssinet, W. Knap, P. Lorenzini, N. Grandjean, J. Massies, C. Skierbiszewski, T. Suski, I. Grzegory, S. Porowski, G. Simin, X. Hu, M. A. Khan, M. S. Shur, R. Gaska, and D. Maude, Appl. Phys. Lett. 77, 2551 (2000).

${ }^{25}$ M. J. Manfra, S. H. Simon, K. W. Baldwin, A. M. Sergent, K. W. West, R. J. Molnar, and J. Caissie, Appl. Phys. Lett. 85, 5278 (2004).

${ }^{26}$ S. Schmult, M. J. Manfra, A. M. Sergent, A. Punnoose, H. T. Chou, D. Goldhaber-Gordon, and R. J. Molnar, Phys. Status Solidi B 243, 1706 (2006).

${ }^{27}$ D. Jena and U. K. Mishra, Phys. Rev. B 66, 241307 (2002).

${ }^{28}$ J. D. Watson, S. Mondal, G. Gardner, G. A. Csáthy, and M. J. Manfra, Phys. Rev. B 85, 165301 (2012).

${ }^{29}$ M. Hirmer, High-Mobility Two-Dimensional Hole Gases in iii-v Semiconductor Heterostructures: Growth and Transport Properties Ph.D. thesis (University of Regensburg, 2013), URN: urn:nbn:de:bvb:355-epub278908.

${ }^{30}$ E. P. De Poortere, Y. P. Shkolnikov, E. Tutuc, S. J. Papadakis, M. Shayegan, E. Palm, and T. Murphy, Appl. Phys. Lett. 80, 1583 (2002).

${ }^{31}$ J. Lok, M. Lynass, W. Dietsche, K. von Klitzing, and M. Hauser, Physica E 22, 94 (2004).

${ }^{32}$ Y. Xie, C. Bell, M. Kim, H. Inoue, Y. Hikita, and H. Y. Hwang, Solid State Commun. 197, 25 (2014).

${ }^{33}$ Y. Chen, F. Trier, T. Wijnands, R. Green, N. Gauquelin, R. Egoavil, D. V. Christensen, G. Koster, M. Huijben, N. Bovet, S. Macke, F. He, N. H. Andersen, G. E. D. K. Prawiroatmodjo, T. S. Jespersen, J. A. Sulpizio, M. Honig, S. Linderoth, S. Ilani, J. Verbeeck, G. Van Tendeloo, G. Rijnders, G. A. Sawatsky, and N. Pryds, Nat. Mater. 14, 801 (2015). 